\title{
ARTICLE
}

Clinical Study

\section{Nut consumption and the risk of oesophageal squamous cell carcinoma in the Golestan Cohort Study}

\author{
Maryam Hashemian 1,2,3, Gwen Murphy ${ }^{2}$, Arash Etemadi ${ }^{1,2}$, Hossein Poustchi ${ }^{4}$, Maryam Sharafkhah ${ }^{1}$, Farin Kamangar ${ }^{1,5}$, \\ Akram Pourshams ${ }^{4,6}$, Akbar Fazeltabar Malekshah ${ }^{1}$, Masoud Khoshniaa, ${ }^{6,7}$, Abdolsamad Gharavi ${ }^{6,7}$, Azita Hekmatdoost ${ }^{8}$, Paul J. Brennan ${ }^{9}$, \\ Paolo Boffetta ${ }^{10}$, Sanford M. Dawsey ${ }^{2}$, Christian C. Abnet ${ }^{2}$ and Reza Malekzadeh ${ }^{1,6}$
}

BACKGROUND: Nut consumption has been inversely associated with gastric cancer incidence in US-based studies, but not with oesophageal cancer. However, there is aetiologic heterogeneity, among oesophageal squamous cell carcinoma (ESCC) cases in lowrisk vs. high-risk populations. The objective of this study was to evaluate the association between nut consumption and risk of ESCC in a high-risk population.

METHODS: The Golestan Cohort Study enroled 50,045 participants in Northeastern Iran, between 2004 and 2008 . Intake of peanuts, walnuts and mixed nuts (including seeds) were assessed using a validated food frequency questionnaire at baseline. Cox proportional hazard models were used to estimate hazard ratios (HR) and 95\% confidence intervals for subsequent ESCC adjusted for potential confounders. Non-consumers of nuts were used as the reference category and the consumers were categorised into tertiles.

RESULTS: We accrued 280 incident ESCC cases during 337,983 person-years of follow up. Individuals in the highest tertiles of total nut consumption, and mixed nut consumption were significantly associated with lower risk of developing ESCC compared to nonconsumers $(\mathrm{HR}=0.60,95 \% \mathrm{Cl}=0.39-0.93$, p-trend $=0.02$, and $\mathrm{HR}=0.52,95 \% \mathrm{Cl}=0.32-0.84, \mathrm{p}$ trend $=0.002$, respectively).

CONCLUSIONS: We found a statistically significant inverse association between total nut consumption and the risk of ESCC in this high-risk population.

British Journal of Cancer (2018) 119:176-181; https://doi.org/10.1038/s41416-018-0148-0

\section{INTRODUCTION}

Nuts appear to have beneficial effects on all cause and cancerspecific mortality, ${ }^{1,2}$ and are part of the recommended Mediterranean diet. ${ }^{3}$ A source of polyphenols, vitamins, minerals and fibres, ${ }^{4}$ nuts can also be rich in monounsaturated fatty acids (MUFA) or polyunsaturated fatty acids, depending on the type of nut. ${ }^{4}$ Nuts are hypothesised to protect against cancer development by inhibition of cell proliferation, enzyme regulation, inhibition of oncogenes, induction of tumour suppressor gene expression, and induction of cell differentiation and apoptosis. ${ }^{5}$ Previous studies have demonstrated that nut consumption is associated with a decreased risk of gastric, colorectal, and pancreatic cancers, ${ }^{6,7}$ and cancer-specific mortality. ${ }^{1}$ Although all nuts are nutritious foods, there are some differences in their vitamin, mineral and fatty acid composition. However, the effect of each type of nut has not been investigated before.
Oesophageal cancer is the sixth most common cause of cancer death worldwide. ${ }^{8}$ The tumour has two different subtypes: oesophageal adenocarcinoma and oesophageal squamous cell carcinoma (ESCC). ${ }^{8}$ ESCC is the most common type of oesophageal cancer in the world, representing more than $80 \%$ of all cases. ${ }^{9}$ In a recent study, nut consumption was not associated with the risk of ESCC in a cohort of middle-aged US adults. ${ }^{10}$ However, there is substantial aetiologic heterogeneity in ESCC between lowincidence populations, where smoking and alcohol are the predominant risk factors, and high-incidence populations, where these two agents play a much smaller role. ${ }^{11}$ Dietary factors are also likely to be different in low and high-incidence populations. ${ }^{12}$

Northeastern Iran has high rates of $\mathrm{ESCC}^{13}$ and was first investigated by the International Agency for Research on Cancer and local investigators in the 1960s and 1970s. ${ }^{14,15}$ In the early 2000 s an international collaborative group restarted an oesophageal cancer research programme in the region that is now known

\footnotetext{
${ }^{1}$ Digestive Oncology Research Center, Digestive Diseases Research Institute, Tehran University of Medical Sciences, Tehran, Iran; ${ }^{2}$ Metabolic Epidemiology Branch, Division of

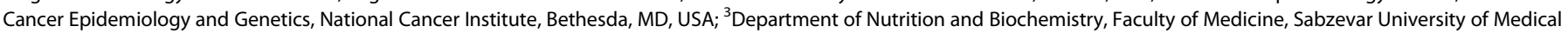

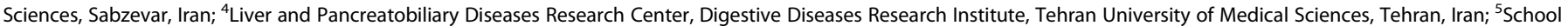

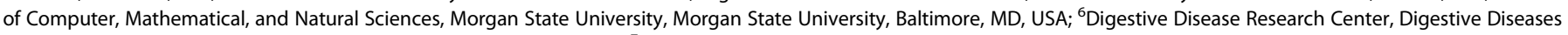
Research Institute, Tehran University of Medical Sciences, Tehran, Iran; ${ }^{7}$ Golestan Research Center of Gastroenterology and Hepatology, Golestan University of Medical Sciences,

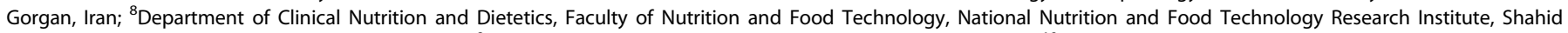

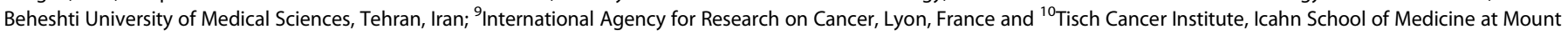
Sinai, New York, NY, USA

Correspondence: Christian C. Abnet (abnetc@nih.gov) or Reza Malekzadeh (Malek@ams.ac.ir)
}

Received: 13 December 2017 Revised: 21 March 2018 Accepted: 23 May 2018

Published online: 28 June 2018 
as Golestan Province. ${ }^{16}$ An inverse association has been reported between nut consumption and total cancer mortality in the Golestan Cohort Study. ${ }^{17}$ The aim of the current study was to evaluate the associations between nut consumption and the risk of ESCC in this same cohort. This study provides data on different types of nuts, including peanuts, walnuts, and mixed nuts (including seeds), which allows us to evaluate the effect of each type of nut on ESCC.

\section{METHODS}

Study population

A detailed description of the study design and methods has been published previously. ${ }^{16}$ In brief, 50,045 adults, aged 40 years old and above, from Golestan Province in northeastern Iran were recruited between January 2004 and June 2008. The total cohort was $42 \%$ male, $80 \%$ rural, $74 \%$ Turkman, and $70 \%$ with no formal education. At baseline, each participant completed a general questionnaire consisting of demographic and risk factor questions and a food frequency questionnaire (FFQ). After enrolment, participants were followed for vital status, major causes of death, incident cancer and cardiovascular disease. For the current analysis, the exclusion criteria were as follow: participants with missing information on nut consumption $(n=872)$, persons with extreme energy intake, defined as more than two interquartile ranges above the 75th percentile or below the 25 th percentile of intake $(n=642)$, and participants who reported history of any cancer except non-melanoma skin cancer $(n=154)$ or had reported renal failure at baseline because they should not consume nuts $(n=93)$. This analysis includes the remaining 48,284 participants. All participants provided written informed consent at baseline.

The study was approved by the Institutional Review Boards of the Digestive Disease Research Institute of Tehran University of Medical Sciences, Iran; the National Cancer Institute $(\mathrm{NCl})$ in the United States; and the International Agency for Research on Cancer (IARC) in France.

\section{Dietary Assessment}

Dietary information was collected using a 116-item semiquantitative FFQ specifically designed for this population, which has been validated against twelve 24-hour dietary recall questionnaires. ${ }^{18}$ Participants were asked about their consumption of common nuts in this population including "peanuts", "walnuts" and "mixed nuts and seeds" (which in this area are typically composed of watermelon seeds, pumpkin seeds, pistachios, almonds, and other tree nuts). Hidden source of nuts and seeds are not common in this population. Subjects reported their frequency of consumption of a given serving size of each food item daily, weekly or monthly during the previous year. Daily intake of each food item was calculated by multiplying the frequency of consumption by the typical portion size and the number of servings per day. For this study, daily intake of each food item was converted to grams. We constructed a new variable "total nut consumption", which summed the consumption of peanuts, walnuts, and mixed nuts and seeds.

\section{Ascertainment of end points}

In our analyses, the primary endpoint was first incident ESCC. At the time of enrolment, all participants were instructed to contact the cohort team in case of any certain conditions like a new major disease or hospitalisation. In addition, participants were contacted by telephone and case review questionnaires were completed once every year. Any hospital admissions or occurrence of disease that had taken place since the previous follow-up contacts were recorded. Reports of cancers, upper GI endoscopy or death were followed by a home or hospital visits to collect all clinical reports, pathology reports and tumour samples (if available). A verbal autopsy was performed for deceased participants. Two internists reviewed all available reports and allocate a disease code for each outcome. In case of any discrepancy, a third expert internist reviewed the documents and made the final decision on the code. Since oesophageal cancer was the most important outcome of the study, all medical or pathology reports of oesophageal cancer were reviewed and verified by an Endpoint Review Committee composed of experts from the Digestive Diseases Research Institute of Tehran University. 239 out of 280 cases were histologically confirmed. Ascertainment of mortality and cancer endpoints has been described in detail elsewhere ${ }^{16}$. During the period of analysis, 369 participants (0.7\%) were lost to follow-up.

\section{Statistical analysis}

Nut consumption was reported in four categories. Since $28 \%$ of participants reported no nut consumption in the FFQ during past 12 months, we made non-consumers the reference group for our analyses, and the consumers were categorised into tertiles using the grams of nuts they consumed per $1000 \mathrm{kcal}$. The median of each category (tertile) was used to assess linear trends. Nut consumption was also evaluated as a continuous variable scaled to 5 grams/day increments (approximately equal to $1 / 4$ cup increments per week). We also investigated ever vs. never nut consumers. In an additional analysis, we categorised nut consumers by quartiles, using the lowest category of consumers as the reference.

Differences in potential risk factors of ESCC in this region were examined across categories of nut consumption. A Cox proportional hazard model was used to estimate hazard ratios (HRs) and $95 \%$ confidence intervals $(95 \% \mathrm{Cls})$. The proportional hazards assumption was tested (and upheld) using Aalen plots and the Schoenfeld residuals test. For this analysis, person-years were calculated as the time from the completion of the baseline FFQ to one of the following events: (1) diagnosis of gastrooesophageal cancer, (2) death, (3) loss to follow-up or (4) the end of follow-up for this analysis (1 November 2016), whichever came first.

Multivariable models were adjusted for known or suspected risk factors for ESCC in this population, including age (years), sex (M/F), BMI ( $<18.5, \geq 18.5, \geq 25, \geq 30 \mathrm{~kg} / \mathrm{m}^{2}$ ), formal education (none, any), ethnicity (non-Turkmen, Turkmen), place of residence (urban, rural), smoking status (pack-year), opium use (nokhod-year, a local unit for opium consumption that weighs about $200 \mathrm{mg}$ ), alcohol drinking (never, ever), physical activity at work (irregular nonintense, regular non-intense, irregular or regular intense), ${ }_{1}^{19}$ wealth score (a composite score including household ownership, house size, appliances, vehicles and other variables associated with wealth, ${ }^{20}$ categorised to tertiles), intake of fruits and vegetables (grams per $1000 \mathrm{Kcal}$ ) and total energy intake. Energy adjustment was conducted using an energy density model based on grams per $1000 \mathrm{Kcal}$ intake, in addition to including energy in the model. $^{21}$ When modelling risks for walnuts and peanuts, we obtained estimates for both from a single model and a joint model (mutual adjustment) to assess the independence of the effects.

We evaluated potential effect modification by age, sex, ethnicity, BMI, smoking status, opium status and alcohol drinking by including a cross-product in the model using likelihood ratio tests. For evaluating interactions among continuous variables, we used medians to categorise them. A sensitivity analysis was performed by excluding the first two years of follow-up to assess whether reverse casualty could be a concern. Other sensitivity models included: excluding participants with BMI less than 18.5 $\mathrm{kg} / \mathrm{m}^{2}$ or more than $35 \mathrm{~kg} / \mathrm{m}^{2}$; excluding participants in the first or last deciles of wealth score; and excluding participants who were tobacco smokers, opium users and/or alcohol drinkers. Statistical analyses were performed using STATA software (version 13, STATA Corp, College Station, TX, USA). Reported $p$-values are two-sided, and $p$-values $<0.05$ were considered statistically significant. 
Table 1. Baseline characteristics of subjects by categories of total nut intake in the Golestan Cohort Study ${ }^{a}$

\begin{tabular}{|c|c|c|c|c|c|}
\hline & $\begin{array}{l}\text { Total population } \\
(N=48,284)\end{array}$ & \multicolumn{4}{|c|}{ Nut intake ${ }^{\mathrm{b}}$ categories $(\mathrm{C})$} \\
\hline Age, years, mean $\pm S D$ & $52.1 \pm 8.9$ & $55.8 \pm 9.4$ & $52.2 \pm 8.7$ & $50.4 \pm 8.1$ & $49.3 \pm 7.7$ \\
\hline $\mathrm{BMI}, \mathrm{kg} / \mathrm{m}^{2}$, mean $\pm \mathrm{SD}$ & $26.7 \pm 5.4$ & $25.5 \pm 5.5$ & $26.6 \pm 5.4$ & $27.1 \pm 5.4$ & $27.5 \pm 5.3$ \\
\hline $\begin{array}{l}\text { Vegetable intake, grams per } \\
1000 \text { kcal, mean } \pm \text { SD }\end{array}$ & $87.2 \pm 38.3$ & $83.5 \pm 40.1$ & $85.6 \pm 36.3$ & $87.5 \pm 36.0$ & $92.6 \pm 39.5$ \\
\hline Sex, male (\%) & 42.3 & 39.9 & 40.7 & 42.9 & 45.2 \\
\hline Place of residence, rural (\%) & 79.7 & 89.6 & 77.3 & 77.1 & 73.5 \\
\hline Ethnicity, Turkmen (\%) & 74 & 68.3 & 72.7 & 76.6 & 79.0 \\
\hline \multicolumn{6}{|l|}{ Physical activity at work } \\
\hline Regular, non-intense (\%) & 26.3 & 16.1 & 25.4 & 29.7 & 35.1 \\
\hline $\begin{array}{l}\text { Regular or irregular, intense } \\
(\%)\end{array}$ & 11.6 & 11.8 & 12.6 & 11.6 & 10.4 \\
\hline \multicolumn{6}{|l|}{ Wealth score } \\
\hline Low (\%) & 32.1 & 43.4 & 33.1 & 27.4 & 22.8 \\
\hline Medium (\%) & 33.6 & 38.0 & 35.1 & 32.4 & 28.5 \\
\hline High (\%) & 34.3 & 18.7 & 31.8 & 40.2 & 48.6 \\
\hline Ever alcohol drinker (\%) & 3.5 & 2.2 & 3.1 & 3.5 & 5.0 \\
\hline Smoking, pack-year & $2.9 \pm 10.1$ & $3.5 \pm 11.7$ & $2.9 \pm 10.2$ & $2.7 \pm 9.0$ & $2.6 \pm 8.7$ \\
\hline Opium use, nokhod-year ${ }^{c}$ & $9.6 \pm 49.7$ & $13.1 \pm 62.6$ & $8.7 \pm 42.9$ & $8.1 \pm 43.8$ & $8.0 \pm 44.5$ \\
\hline
\end{tabular}

AAll covariates were associated with nut consumption with $p<0.001$. ' Intake density (grams per $1000 \mathrm{kcal}$ ), including tree nut, peanut, walnut and seed. ${ }^{\mathrm{C}} \mathrm{A}$ local unit for opium consumption that weighs about $200 \mathrm{mg}$

\section{RESULTS}

$72 \%$ of the participants reported consuming nuts. The median (IQR) intake of total nuts, mixed nuts and seeds, peanuts and walnuts were 1.89 (0.69-4.46), $1.33(0.74-3.53), 0.83(0.34-1.79)$ and 0.22 (0.09-0.71) $\mathrm{g} /$ day among nut consumers, respectively. Table 1 shows the baseline characteristics of participants by categories of total nut intake per $1000 \mathrm{kcal}$. Nut consumers were more likely to be younger compared to those who reported never consuming nuts. Further, they were more likely to have a higher wealth score, live in urban areas, and consume more fruits and vegetables, and they were more likely to be male, Turkman, have a higher BMI, and have formal education. They were also more likely to drink alcohol and less likely to smoke tobacco or opium. Mix nut and seed consumer, peanut consumer and walnut consumer had the same pattern for these confounders (Supplementary Table 1).

A total of 280 participants developed ESCC during the study period, with a median follow up of 9 years. Table 2 shows the associations between nut consumption and risk of incident ESCC. The highest tertile of total nut consumption was associated with lower risk of developing ESCC compared to non-consumers (adjusted $\mathrm{HR} \mathrm{C} 3_{\mathrm{vs}} \mathrm{CO}=0.60,95 \% \mathrm{Cl}=0.39-0.93, p$-trend $=0.02$ ). $\mathrm{A}$ similar inverse association was observed between mixed nut and seed consumption and risk of ESCC (adjusted HR C3 ${ }_{\text {vs }} \mathrm{CO}=0.52$, $95 \% \mathrm{Cl}=0.32-0.84, p$ trend $=0.002$ ). By contrast, neither peanut or walnut consumption was significantly associated with risk of ESCC (adjusted HR $\mathrm{C}_{\mathrm{vs}} \mathrm{CO}=0.80,95 \% \mathrm{Cl}=0.51-1.24$ and adjusted $\mathrm{HR}$ $\mathrm{C}_{\text {vs }} \mathrm{CO}=0.71,95 \% \mathrm{Cl}=0.45-1.14$, respectively (Table 2 ).

The ever vs. never (total nut) consumers had a decreased risk of developing ESCC (adjusted $\mathrm{HR}=0.62,95 \% \mathrm{Cl}=0.42-0.92$ ).
However, for the individual categories of mixed nut and seed, peanut or walnut ever vs. never consumption, the inverse associations were not significant (adjusted $\mathrm{HR}=0.80,95 \% \mathrm{Cl}=$ $0.61-1.05$, adjusted $\mathrm{HR}=0.99,95 \% \mathrm{Cl}=0.77-1.30$, adjusted $\mathrm{HR}=$ $0.80,95 \% \mathrm{Cl}=0.61-1.05$, respectively).

Modelling nut consumption as a continuous variable, we observed a statistically significant $29 \%$ decrease in ESCC risk for each $5 \mathrm{~g}$ of total nuts consumed per day (Table 2). An inverse association between $5 \mathrm{~g} /$ day increases in peanut or walnut consumption and risk of ESCC did not reach statistical significance (Table 2). Limiting the analysis to nut consumers, the highest quartile of total nut consumption was associated with lower risk of developing ESCC compared to the lowest quartile (adjusted HR $\mathrm{Q}_{\text {vs }} \mathrm{Q} 1=0.59,95 \% \mathrm{Cl}=0.35-0.99$ ).

Mutual adjustment for peanuts or walnuts did not substantially alter risk estimates (data not shown). We found no evidence of effect modification by age, sex, ethnicity, BMl, smoking status, opium status and alcohol drinking ( $p>0.05$ for all analyses). Table 3 shows the results of sensitivity analyses by excluding the first two years of follow-up; those with extreme BMl; those with extreme wealth scores; or those who were smokers, opium users and/or alcohol drinkers. None of these models suggested meaningful differences in the overall results.

\section{DISCUSSION}

In a population with a high ESCC incidence, we found an inverse association between total nut consumption and the risk of ESCC. Similarly, we found an inverse association with mixed nuts and 
Table 2. Crude and adjusted Hazard ratios of incident oesophageal squamous cell carcinoma, by nut intake $(N=48,284)$

\begin{tabular}{|c|c|c|c|c|c|c|}
\hline & \multicolumn{5}{|l|}{ Categories of intake ${ }^{b}$} & \multirow{2}{*}{$\begin{array}{l}\text { Continuous HR per } 5 \mathrm{~g} / 1000 \mathrm{kcal} / \text { day } \\
\text { increased intake }\end{array}$} \\
\hline & No nut consumption & Tertile 1 & Tertile 2 & Tertile 3 & $\overline{p \text {-trend }}{ }^{c}$ & \\
\hline $\begin{array}{l}\text { Mean intake }(\mathrm{g} / 1000 \\
\mathrm{kcal}) \pm \mathrm{SD}\end{array}$ & - & $0.21 \pm 0.13$ & $0.92 \pm 0.29$ & $3.84 \pm 3.23$ & & \\
\hline Person years & 114,663 & 106,297 & 107,322 & 111,456 & & \\
\hline $\mathrm{HR},(95 \% \mathrm{Cl})^{\mathrm{e}}$ & 1 & $0.88(0.66-1.19)$ & $0.84(0.61,1.17)$ & $0.49(0.32-0.73)$ & 0.001 & $0.64(0.50-0.81)$ \\
\hline$H R,(95 \% \mathrm{Cl})^{f}$ & 1 & $1.02(0.75-1.39)$ & $1.03(0.73,1.44)$ & $0.60(0.39-0.93)$ & 0.02 & $0.71(0.55-0.91)$ \\
\hline \multicolumn{7}{|l|}{ Mixed nuts and seeds } \\
\hline $\begin{array}{l}\text { Mean intake }(\mathrm{g} / 1000 \\
\mathrm{kcal}) \pm \mathrm{SD}\end{array}$ & - & $0.16 \pm 0.09$ & $0.61 \pm 0.19$ & $2.90 \pm 2.73$ & & \\
\hline $\mathrm{HR},(95 \% \mathrm{Cl})^{\mathrm{e}}$ & 1 & $0.91(0.66-1.25)$ & $0.53(0.34-0.81)$ & $0.47(0.29-0.74)$ & $<0.001$ & $0.54(0.36-0.80)$ \\
\hline $\mathrm{HR},(95 \% \mathrm{Cl})^{\mathrm{f}}$ & 1 & $1.21(0.87-1.68)$ & $0.58(0.37-0.91)$ & $0.52(0.32-0.84)$ & 0.002 & $0.57(0.38-0.86)$ \\
\hline \multicolumn{7}{|l|}{ Peanuts } \\
\hline $\begin{array}{l}\text { Mean intake (g/ } 1000 \\
\text { kcal) } \pm \text { SD }\end{array}$ & - & $0.12 \pm 0.05$ & $0.41 \pm 0.14$ & $1.94 \pm 2.10$ & & \\
\hline Person years & 221830 & 70670 & 73386 & 74385 & & \\
\hline Cases, $n$ & 177 & 36 & 41 & 26 & & \\
\hline $\mathrm{HR},(95 \% \mathrm{Cl})^{\mathrm{d}}$ & 1 & $0.63(0.44-0.91)$ & $0.71(50-0.99)$ & $0.43(0.28-0.65)$ & $<0.001$ & $0.48(0.30-0.78)$ \\
\hline $\mathrm{HR},(95 \% \mathrm{Cl})^{\mathrm{e}}$ & 1 & $0.83(0.58-1.20)$ & $1.03(0.73-1.46)$ & $0.66(0.43-1.01)$ & 0.001 & $0.73(0.48-1.09)$ \\
\hline $\mathrm{HR},(95 \% \mathrm{Cl})^{\mathrm{f}}$ & 1 & $0.97(0.67-1.41)$ & $1.21(0.84-1.72)$ & $0.80(0.51-1.24)$ & 0.02 & $0.85(0.59-1.24)$ \\
\hline \multicolumn{7}{|l|}{ Walnuts } \\
\hline \multicolumn{7}{|c|}{$\begin{array}{l}\left.{ }^{a} \text { Intake density (g per } 1000 \mathrm{kcal}\right) .{ }^{\mathrm{b}} \text { The categories of intake were defined separately for each nut category. }{ }^{\mathrm{c}} \text { The test for trend used ordinal models with tertile } \\
\text { mid-points as values. }{ }^{\mathrm{d} C r u d e} \text { models. }{ }^{\mathrm{e}} \text { Adjusted for age (years) and sex. }{ }^{\mathrm{f}} \text { Adjusted for age (years), sex, place of residence (urban, rural), smoking (pack-year), } \\
\text { opium user (nokhod-year, a local unit for opium consumption that weighs about } 200 \mathrm{mg} \text { ), wealth score (low, medium, high), ethnicity (non-Turkmen, } \\
\text { Turkmen), body mass index }\left(<18.5, \geq 18.5, \geq 25, \geq 30 \mathrm{~kg} / \mathrm{m}^{2}\right) \text {, education (no formal education, formal education), physical activity (irregular non-intense, regular } \\
\text { non-intense, regular or irregular intense), fruits intake }(\mathrm{g} / 1000 \mathrm{kcal}) \text { and vegetables intake (g/1000 kcal); HRs ( } 95 \% \mathrm{Cl} \text { ) were calculated using Cox regression } \\
\text { models }\end{array}$} \\
\hline
\end{tabular}

seeds consumption. However, the risk of ESCC was not associated with reported walnut or peanut consumption. To our knowledge, this is the first prospective study assessing the association between consumption of different types of nut and the risk of ESCC.

An inverse association between total nut consumption and cancer mortality was previously noted in a meta-analysis. ${ }^{1}$ In a previous study in this population in Iran, nut consumption was associated with decreased risk of total mortality and cancer-related mortality. ${ }^{17}$ Further, our current results are consistent with the findings in other types of cancers including colon cancer, lung cancer and pancreatic cancer. ${ }^{7,22}$ By contrast, a recent study in a US cohort showed no association between nut and seed consumption with ESCC risk. ${ }^{10}$ Such a discrepancy between the significance of risk factors in low-risk and high-risk populations has been reported before for smoking, alcohol and sex. ${ }^{11}$ This aetiologic heterogeneity of ESCC is remarkable, but the reason is not clear yet. ${ }^{11}$
We observed a statistically significant inverse association between mixed nut and seed consumption and the risk of ESCC; we also found point estimates $<1$ but no significant association between peanut or walnut consumption and risk of ESCC. In Golestan, where we undertook our study, mixed nuts and seeds would usually include watermelon seeds, pumpkin seeds, pistachios and almonds. The lack of a significant effect for peanut or walnut consumption could be due to lower intake of peanuts and walnuts relative to mixed nuts and seeds in this population. However, the difference could also be explained by the different compositions of different types of nuts. Although all nuts are nutritious foods, there are some differences in their vitamin, mineral and fatty acid composition (Table 4). Among nuts and seeds, watermelon and pumpkin seeds are the best source of zinc (containing about three times more than in peanuts or walnuts). ${ }^{23}$ Zinc intake is inversely associated with the risk of ESCC. ${ }^{19,} 24,25$ Zinc is necessary for immune function, and for transcription 
factors which control cell proliferation and signalling pathways. ${ }^{26,}$

27 Almonds are the best source of calcium and riboflavin among nuts (containing three times more calcium and eight times more riboflavin than peanuts or walnuts). ${ }^{23}$ Both calcium intake and riboflavin status have previously been shown to be inversely associated with ESCC risk. ${ }^{19,28}$ Previous studies showed that calcium suppresses the cell cycle, and promotes apoptosis, ${ }^{29,} 30$ and riboflavin deficiency suppresses immune functions and may fail to control chronic inflammation. ${ }^{31}$ Almonds are also a good source of MUFAs, which have anti-inflammatory effects. ${ }^{23}$ Peanuts are source of folate, and folate has inverse association with risk of ESCC. $^{32}$ Folate deficiency may decrease the level of Sadenosylmethionine and consequently cause DNA hypomethylation and cancer. ${ }^{32}$ Eating a variety of nuts and seeds could provide an excellent source of all of these nutrients in comparison with not eating nuts or eating only one specific type of nut, and greater weight should be placed upon the consumption of watermelon and pumpkin seeds in Iran.

This study has several strengths. The Golestan Cohort is a prospective study, in a high-risk population for oesophageal cancer, with an excellent follow-up rate. Because nut consumption

Table 3. Sensitivity analyses of the association of increasing $5 \mathrm{~g}$ per day of total nut intake ${ }^{a}$ and risk of ESCC

\begin{tabular}{|c|c|c|}
\hline Exclusion & $\mathrm{HR}^{\mathrm{b}}(95 \% \mathrm{Cl})$ & $P$ for trend \\
\hline No exclusions & $0.71(0.56-0.91)$ & 0.03 \\
\hline First 2 years of follow up & $0.71(0.56-0.92)$ & 0.04 \\
\hline Participants with extreme BMI $\mathbf{c}^{\mathbf{C}}$ & $0.74(0.58-0.94)$ & 0.04 \\
\hline Participants with extreme wealth score ${ }^{d}$ & $0.75(0.59-0.97)$ & 0.06 \\
\hline $\begin{array}{l}\text { Smokers, opium users and/or alcohol } \\
\text { drinkers }\end{array}$ & $0.69(0.51-0.95)$ & 0.04 \\
\hline \multicolumn{3}{|c|}{$\begin{array}{l}\text { ancluding tree nut, peanut, walnut and seed. }{ }^{b} \text { Adjusted for age (years), sex } \\
\text { (M, F), place of residence (urban, rural), smoking (pack-year), opium user } \\
\text { (nokhod-year, a local unit for opium consumption that weighs about } 200 \\
\text { mg), wealth score (low, medium, high), ethnicity (non-Turkmen, Turkmen), } \\
\text { body mass index ( }<18.5, \geq 18.5, \geq 25, \geq 30 \text { ), education (no formal, formal } \\
\text { education), physical activity (Irregular non-intense, Regular non-intense, } \\
\text { regular or irregular intense), fruits intake (g/d) and vegetables intake (g/d); } \\
\text { HRs ( } 95 \% \text { Cl) were calculated using Cox regression models. }{ }^{\mathrm{C}} \mathrm{BMI}<18.5 \text { or } \\
\text { BMI }>35^{\mathrm{d}} \text { The first and last deciles of wealth score }\end{array}$} \\
\hline
\end{tabular}

is high in this population, it was assessed using three separate questions (about peanuts, walnuts, and mixed nuts and seeds), in contrast to other studies, many of which used a single question to assess the consumption of nuts. ${ }^{7}$

Our study also has several limitations. One limitation is that some degree of measurement error is inevitable when analysing self-reported intake of foods recorded by an FFQ. Nut consumption was significantly associated with higher socioeconomic status, meaning that nut consumers may have had healthier diets because of their socioeconomic status, and this may partially explain their decreased risk of oesophageal cancer. Adjusting our analysis for wealth score, education, intake of fruits and vegetables and other known risk factors did significantly reduce the strength of all associations. Considering the difference between the crude and adjusted HRs, we cannot exclude the possibility of residual confounding. We were not able to investigate the influence of preparation method or salted vs. unsalted nuts. We also cannot conclude a causal relationship from this observational study.

In conclusion, we found an inverse association between total nut consumption and the risk of ESCC in this large Iranian cohort. This association needs to be confirmed in future studies, along with additional research on the association between nut consumption and risk of total mortality, specific causes of mortality, and incidence of other cancers to inform public health recommendations. We also suggest comparing the effect of nuts and seeds in future studies where the data are available.

\section{ACKNOWLEDGEMENTS}

We express their gratitude to the study participants, to the nutritionists who completed questionnaires and to the Golestan cohort team for their excellent technical support.

\section{AUTHOR CONTRIBUTIONS}

$A P, P J B, P B, S M D, C C A$ and RM designed the study. HP, MK and AG conducted research. $M H, M S$ and $G M$ analysed the data; AFM obtained and cleaned FFQ data. $\mathrm{MH}$ drafted the manuscript; $\mathrm{AE}, \mathrm{AH}$ and $\mathrm{FK}$ critically revised the manuscript. CCA and RM had primary responsibility for final content. All authors had access to data, have read, and approved the final manuscript.

\section{ADDITIONAL INFORMATION}

Supplementary information is available for this paper at https://doi.org/10.1038/ s41416-018-0148-0.

\begin{tabular}{|c|c|c|c|c|c|c|}
\hline Energy (kcal) & 567 & 654 & 579 & 559 & 557 & 560 \\
\hline Iron (mg) & 5 & 3 & 4 & 9 & 7.3 & 4 \\
\hline Magnesium (mg) & 168 & 158 & 270 & 592 & 515 & 121 \\
\hline Riboflavin (mg) & 0.14 & 0.15 & 1.14 & 0.15 & 0.15 & 0.16 \\
\hline Niacin (mg) & 12.1 & 1.1 & 3.6 & 5.0 & 3.5 & 1.3 \\
\hline Vitamin B6 (mg) & 0.3 & 0.5 & 0.1 & 0.1 & 0.1 & 1.7 \\
\hline Folate $(\mu \mathrm{g})$ & 240 & 98 & 44 & 58 & 58 & 51 \\
\hline Saturated fat (g) & 6 & 6 & 4 & 9 & 10 & 6 \\
\hline
\end{tabular}


Competing interests: The authors declare no competing interests.

Funding: This work was supported by Tehran University of Medical Sciences; the Intramural Research Program of the US National Cancer Institute at the National Institutes of Health; Cancer Research UK [grant number 07CN120-B]; and through various collaborative research agreements with the International Agency for Research on Cancer.

Ethics: The study was approved by the Institutional Review Boards of the Digestive Disease Research Institute of Tehran University of Medical Sciences, Iran; the National Cancer Institute $(\mathrm{NCl})$ in the United States; and the International Agency for Research on Cancer (IARC) in France. Informed consent All participants provided written informed consent at baseline.

Note: This work is published under the standard license to publish agreement. After 12 months the work will become freely available and the license terms will switch to a Creative Commons Attribution 4.0 International (CC BY 4.0).

\section{REFERENCES}

1. Grosso, G. et al. Nut consumption on all-cause, cardiovascular, and cancer mortality risk: a systematic review and meta-analysis of epidemiologic studies. Am. J. Clin. Nutr. 101, 783-793 (2015).

2. van den Brandt, P. A. \& Schouten, L. J. Relationship of tree nut, peanut and peanut butter intake with total and cause-specific mortality: a cohort study and meta-analysis. Int. J. Epidemiol. 44, 1038-1049 (2015).

3. Willett, W. C. et al. Mediterranean diet pyramid: a cultural model for healthy eating. Am. J. Clin. Nutr. 61 (6 Suppl), 1402S-1406SS (1995).

4. Kris-Etherton, P. M. et al. Bioactive compounds in foods: their role in the prevention of cardiovascular disease and cancer. Am. J. Med. 113, 71-88 (2002).

5. Falasca M., Casari I., Maffucci T. Cancer chemoprevention with nuts. J. Natl Cancer Inst. 106, pii: dju238 (2014).

6. Grosso, G. \& Estruch, R. Nut consumption and age-related disease. Maturitas $\mathbf{8 4}$ 11-16 (2016).

7. Lee, J. T. et al. Nut consumption and lung cancer risk: results from two large observational studies. Cancer Epidemiol. Biomark. Prev. 26, 826-836 (2017).

8. Fitzmaurice, C. et al. Global, regional, and national cancer incidence, mortality, years of life lost, years lived with disability, and disability-adjusted life-years for 32 cancer groups, 1990 to 2015: a systematic analysis for the global burden of disease study. JAMA Oncol. 3, 524-548 (2017).

9. Arnold, M., Soerjomataram, I., Ferlay, J. \& Forman, D. Global incidence of oesophageal cancer by histological subtype in 2012. Gut 64, 381-387 (2015).

10. Hashemian, M. et al. Nut and peanut butter consumption and the risk of esophageal and gastric cancer subtypes. Am. J. Clin. Nutr. 106, 858-864 (2017).

11. Murphy, G. et al. International cancer seminars: a focus on esophageal squamous cell carcinoma. Ann. Oncol. 28, 2086-2093 (2017).

12. Abnet, C. C., Arnold, M. \& Wei, W. Q. Epidemiology of esophageal squamous cell carcinoma. Gastroenterology 154, 360-373 (2018).
13. Islami, F. et al. Oesophageal cancer in Golestan Province, a high-incidence area in northern Iran - a review. Eur. J. Cancer 45, 3156-3165 (2009).

14. Kmet, J. \& Mahboubi, E. Esophageal cancer in the Caspian littoral of Iran: initial studies. Science 175, 846-853 (1972).

15. Mahboubi, E. et al. Oesophageal cancer studies in the Caspian Littoral of Iran: the Caspian cancer registry. Br. J. Cancer 28, 197-214 (1973).

16. Pourshams, A. et al. Cohort profile: The Golestan Cohort Study--a prospective study of oesophageal cancer in northern Iran. Int. J. Epidemiol. 39, 52-59 (2010)

17. Eslamparast, T. et al. Nut consumption and total and cause-specific mortality: results from the Golestan Cohort Study. Int. J. Epidemiol. 46, 75-85 (2017).

18. Malekshah, A. F. et al. Validity and reliability of a new food frequency questionnaire compared to $24 \mathrm{~h}$ recalls and biochemical measurements: pilot phase of Golestan cohort study of esophageal cancer. Eur. J. Clin. Nutr. 60, 971-977 (2006).

19. Hashemian, M. et al. Dietary intake of minerals and risk of esophageal squamous cell carcinoma: results from the Golestan Cohort Study. Am. J. Clin. Nutr. 102, 102-108 (2015).

20. Islami, F. et al. Socio-economic status and oesophageal cancer: results from a population-based case-control study in a high-risk area. Int. J. Epidemiol. 38, 978-988 (2009).

21. Willett, W. C., Howe, G. R. \& Kushi, L. H. Adjustment for total energy intake in epidemiologic studies. Am. J. Clin. Nutr. 65(4 Suppl), 1220S-1228SS (1997).

22. Wu, L. et al. Nut consumption and risk of cancer and type 2 diabetes: a systematic review and meta-analysis. Nutr. Rev. 73, 409-425 (2015).

23. US Department of Agriculture ARS, Nutrient Data Laboratory. USDA National Nutrient Database for Standard Reference, Release 28. Version Current: September 2015, slightly revised May 2016. Internet: /nea/bhnrc/ndl.

24. Abnet, C. C. et al. Zinc concentration in esophageal biopsy specimens measured by $\mathrm{x}$-ray fluorescence and esophageal cancer risk. J. Natl. Cancer Inst. 97, 301-306 (2005).

25. Hashemian, M. et al. Systematic review of zinc biomarkers and esophageal cancer risk. Middle East J. Dig. Dis. 6, 177-185 (2014).

26. Fong, L. Y., Li, J. X., Farber, J. L. \& Magee, P. N. Cell proliferation and esophageal carcinogenesis in the zinc-deficient rat. Carcinogenesis 17, 1841-1848 (1996).

27. Liu, C. G. et al. Modulation of gene expression in precancerous rat esophagus by dietary zinc deficit and replenishment. Cancer Res. 65, 7790-7799 (2005).

28. Ren, J. et al. Prospective study of serum B vitamins levels and oesophageal and gastric cancers in China. Sci. Rep. 6, 35281 (2016).

29. Lipkin, M. Application of intermediate biomarkers to studies of cancer prevention in the gastrointestinal tract: introduction and perspective. Am. J. Clin. Nutr. 54(1 Suppl), 188S-192SS (1991).

30. Lamprecht, S. A. \& Lipkin, M. Chemoprevention of colon cancer by calcium vitamin D and folate: molecular mechanisms. Nat. Rev. Cancer 3, 601-614 (2003).

31. WCRF/AICR. Food, Nutrition, Physical Activity, and the Prevention of Cancer: a Global Perspective. (Washington DC, AICR, 2007)

32. Galeone, $\mathrm{C}$. et al. Folate intake and squamous-cell carcinoma of the oesophagus in Italian and Swiss men. Ann. Oncol. 17, 521-525 (2006). 